\title{
Editorial
}

\section{Biodegradable Polymers for Medical Applications}

\author{
Anezka Lengalova, ${ }^{1}$ Alenka Vesel, ${ }^{2}$ Yakai Feng, ${ }^{3}$ and Vitor Sencadas ${ }^{4}$ \\ ${ }^{1}$ Centre of Polymer Systems, Tomas Bata University in Zlín, 76001 Zlín, Czech Republic \\ ${ }^{2}$ Jozef Stefan Institute, SI-1000 Ljubljana, Slovenia \\ ${ }^{3}$ School of Chemical Engineering and Technology, Tianjin University, Tianjin 300072, China \\ ${ }^{4}$ School of Mechanical, Materials and Mechatronics Engineering, University of Wollongong, Wollongong, NSW 2522, Australia
}

Correspondence should be addressed to Anezka Lengalova; lengalova@fhs.utb.cz

Received 5 April 2016; Accepted 6 April 2016

Copyright (C) 2016 Anezka Lengalova et al. This is an open access article distributed under the Creative Commons Attribution License, which permits unrestricted use, distribution, and reproduction in any medium, provided the original work is properly cited.

Biodegradable polymers have a long history which, however, is difficult to be traced as some of them are natural products. The concept of synthetic biodegradable polymers, the topic of this special issue, was introduced in the 1980s. Since then the field has experienced a steady and stable growth as its outcomes are potentially relevant to the majority of population. This interdisciplinary field encompasses elements of materials science, biology, chemistry, medicine, tissue engineering, and others.

The advantage of polymers in this area is that they can be tailored from the viewpoint of their chemical, physical, and surface properties, so as to enable good cell adhesion and proliferation in vivo, maintenance of their properties for a given time, and then degradation with no harmful effects in the body. They are suitable for a variety of applications, but their priority application is in tissue engineering and drug delivery. This reflects also in the present special issue; the articles cover various views on biodegradable polymers.

The paper "Cell Adhesion on Polycaprolactone Modified by Plasma Treatment" shows how plasma treatment of polycaprolactone fibrous membrane surface can affect adhesion and proliferation of human cells. Thus this material with improved biocompatibility can be more effectively used as scaffolds in the body.

Scaffolds are also dealt with in the paper "Designing of Collagen Based Poly(3-hydroxybutyrate-co-4-hydroxybutyrate) Scaffolds for Tissue Engineering." With the aim of enhancing the hydrophilicity for application of $\mathrm{P}(3 \mathrm{HB}-$ co-4HB) copolymer in tissue engineering, collagen was incorporated in the surface. At the optimum conditions the material showed significant cell adhesion and proliferation on the surface, so its ability to be used for biopurposes increased. Hence surface modification by blending biomacromolecules proved to enhance the biocompatibility of the original polymeric material.

Similarly to the first one, also the paper "Haemostatic Response of Polyethylene Terephthalate Treated by Oxygen and Nitrogen Plasma Afterglows" is connected with plasma treatment, in this case plasma afterglow. Afterglow was used to alter haemostatic response of PET polymer and binding of anticoagulant heparin. The study shows improved biocompatibility of plasma-treated PET with and without heparin coating in contact with fresh blood. Although it was still lower than that of the PET with covalently bonded heparin, it is another promising direction of research.

The fact that hydrogels are materials with a great potential is demonstrated in two papers. One of them, "Direct Synthesis of Hyperbranched Poly(acrylic acid-co-3hydroxypropionate)," presents the stage of the material preparation. The produced hyperbranched PAcHP exhibits hydrogel properties and thus can be considered as a biodegradable polymer matrix for drug delivery or hydrogel scaffold for tissue engineering applications. The other paper, "Effect of Sodium Salicylate on the Viscoelastic Properties and Stability of Polyacrylate-Based Hydrogels for Medical Applications," goes a step further; it studies long term stability, an important aspect for practical medical use, of polyacrylate-based hydrogel. The presence of sodium salicylate noticeably influences hydrogel neutralization, 
rheological and antimicrobial properties, and has a positive effect on aging.

The paper "Antiepileptic Effects of Lacosamide Loaded Polymers Implanted Subdurally in GAERS" concentrates on the possibility of using targeted drug delivery for the treatment of long-lasting seizure remission from epilepsy, which can be a hope for patients in future. The biodegradable polymer involved here was poly(D,L-lactide-co-glycolide) in the form of electrospun mats and it was loaded with lacosamide. This was implanted with skull electrodes to a special strain of rats. In comparison with blanks the material showed a partial sustained antiepileptic effect, which is a good start for further development of the method.

We hope that the readers of this special issue will consider it not only as a source of information, but also as an inspiration for their further research, for example, in cooperation of researchers from different institutions or even different countries.

\title{
Acknowledgments
}

We would like to thank all authors who sent their papers for the review in this special issue. We paid large attention to the selection, so not all of them could be published, for either the reasons of unsuitable topics, or unsatisfactory quality. However, all the effort is appreciated.

\author{
Anezka Lengalova \\ Alenka Vesel \\ Yakai Feng \\ Vitor Sencadas
}



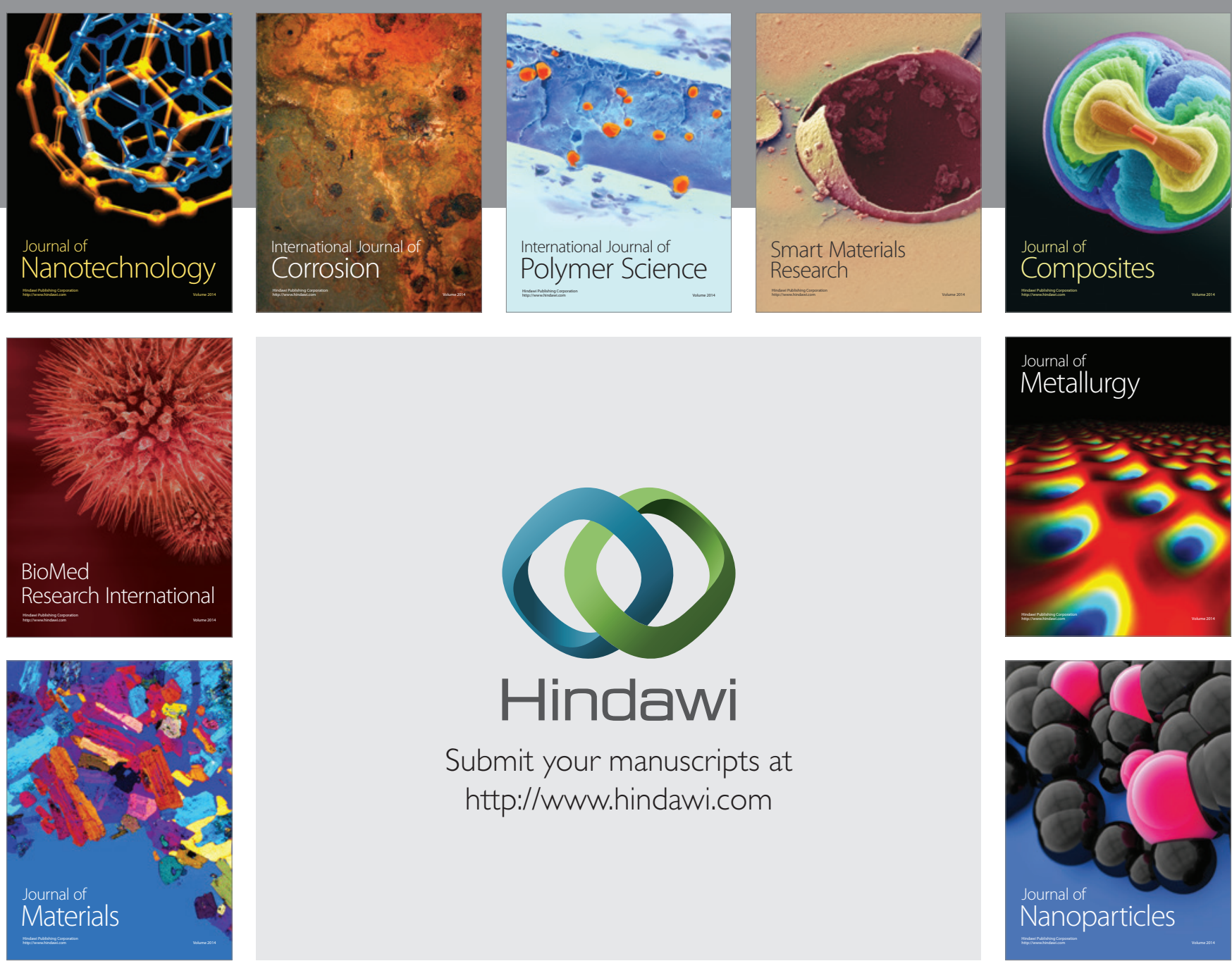

\section{Hindawi}

Submit your manuscripts at

http://www.hindawi.com

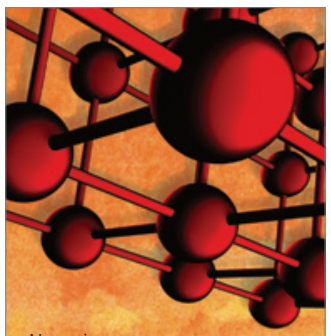

Materials Science and Engineering
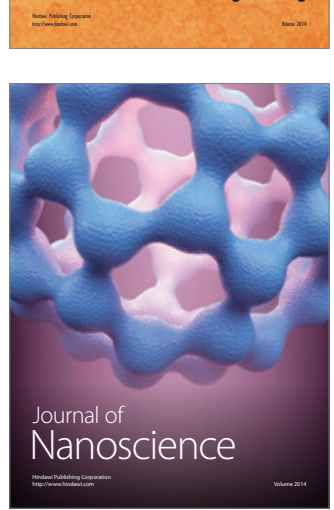
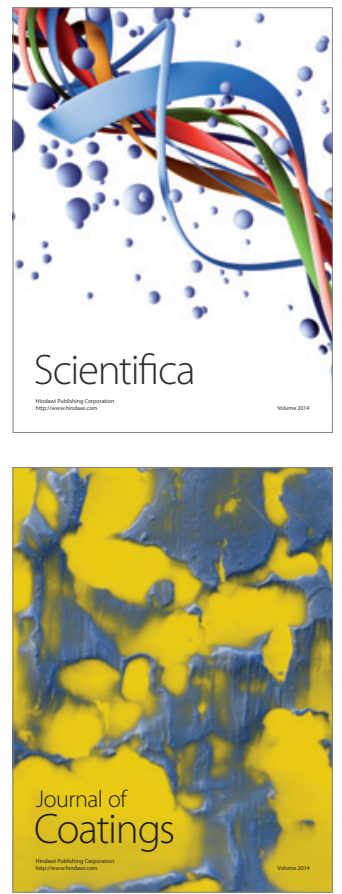
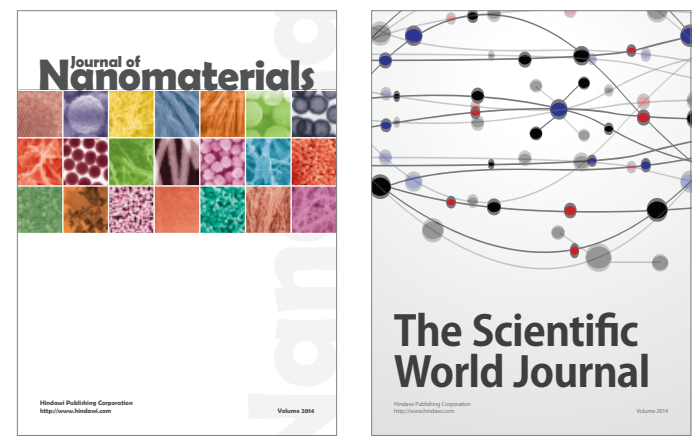

The Scientific World Journal
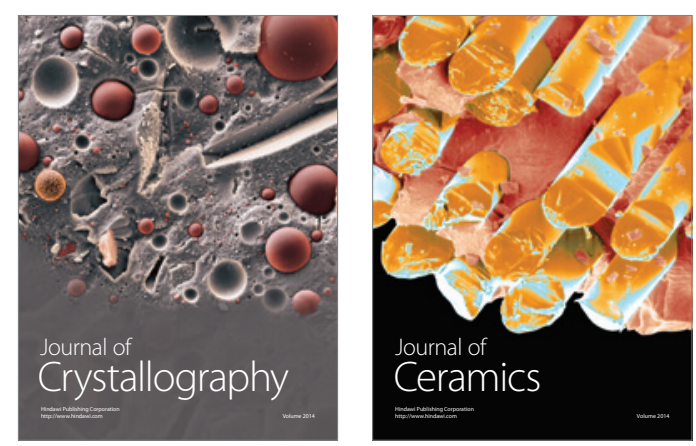
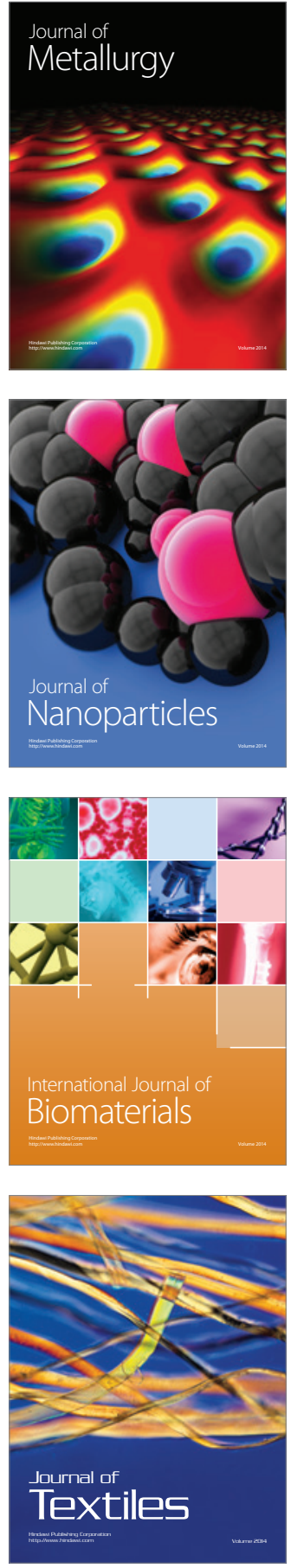